\title{
ON THE FINITE MEASURES ON THE CLOSED SUBSPACES OF A HILBERT SPACE
}

\author{
C. C. BROWN
}

In his article Measures on the closed subspaces of a Hilbert space [1], A. M. Gleason proves the following theorem.

TheOREM 4.1. Let $\mu$ be a measure on the closed subspaces of a separable (real or complex) Hilbert space $H$ of dimension at least three. There exists a nonnegative operator $J$ with trace $(J)<\infty$ such that for every closed subspace $E \subset H, \mu(E)=$ trace $\left(J P_{E}\right)$ where $P_{E}$ is the projection of $H$ onto $E$.

For the purposes of quantum theory it is desirable to have at least the following theorem.

THEOREM I. Let $\mu$ be a finite measure on the projections of the separable complex Hilbert space $H$. Then there exists exactly one nonnegative operator $J$ with trace $(J)<\infty$ such that $\mu(P)=$ trace $(J P)$ for all projections $P$.

By the term "finite measure on the projections" we mean a finite nonnegative function $\mu$ on the projections of $H$ having the property: If $P_{1}, P_{2}, P_{3}, \cdots$ is a sequence of mutually orthogonal projections, then

$$
\mu\left(\sum_{i} P_{i}\right)=\sum_{i} \mu\left(P_{i}\right)
$$

For the case where the dimension of $H$ is greater than two, this theorem is already contained in that of Gleason. Gleason's proof of Theorem 4.1 is rather involved, however, and it is the purpose of this paper to supply a proof of Theorem I which is in principle simple.

We begin with a few remarks already proved in a simple manner in a previous paper of the author [2].

Let $H$ be a separable complex Hilbert space and $W$ the set of nonnegative operators $J$ with trace $(J)<\infty$.

Theorem. If $f(\cdot)$ is a nonnegative finite valued linear form on $W$, then there exists exactly one bounded nonnegative operator $A_{f}$ on $H$ with $f(J)=$ trace $\left(J A_{f}\right)$ for all $J$ in $W$; and the mapping $f \rightarrow A_{f}$ is a one to one linear isomorphism onto the bounded nonnegative operators in $H$.

Received by the editors January 3, 1967. 
REMARK. As was shown in [2], any finitely valued nonnegative linear form $f$ on $W$ is automatically normal (i.e., $\sup f\left(A_{i}\right)=f\left(\sup A_{i}\right)$ for any nondecreasing sequence $\left\{A_{i}\right\}$ in $W$ for which $\sup A_{i}$ exists).

We denote by $\bar{W}$ the class of all finite measures $\mu$ on the projections of $H$. It is easily seen that $W \subset \bar{W}$ since, for each $J \in W$, we can define $J(P)=\operatorname{trace}(J P)$ which is a finite measure.

If $\bar{f}$ is any finite nonnegative linear form on $\bar{W}$, then $\bar{f}$ also defines a nonnegative finite linear form on $W$ and there exists exactly one bounded nonnegative linear operator $A_{\bar{f}}$ such that $\bar{f}(J)$ $=$ trace $\left(J A_{\bar{f}}\right)(J \in W)$ and the mapping $\Phi: \bar{f} \rightarrow A_{\bar{f}}$ is linear and one to one.

Lemma. The mapping $\Phi: \bar{f} \rightarrow A_{\bar{f}}$ is onto the class of all nonnegative bounded operators in $H$.

Proof. Let $A$ be a nonnegative bounded operator. By the spectral theorem, $A$ is the supremum of an increasing sequence of operators $A_{n}$ of the form $A_{n}=\sum_{1} \lambda_{i}^{n} P_{i}^{n}$ where, for each $n$, the $P_{i}^{n}$ are mutually orthogonal projections of the spectral family of $A$ and where the $\lambda_{i}^{n}$ are nonnegative and uniformally bounded by $\|A\|$. We define

$$
\bar{f}_{A_{n}}(\mu)=\sum_{i} \lambda_{i}^{n} \mu\left(P_{i}^{n}\right)
$$

for all $\mu \in \bar{W} . \bar{f}_{A_{n}}(\cdot)$ is a nonnegative linear form on $\bar{W}$. We have

$$
\bar{f}_{A_{n}}(\mu) \leqq\|A\| \sum_{i} \mu\left(P_{i}^{n}\right)=\|A\|_{\mu}\left(\sum_{i} P_{i}^{n}\right) \leqq\|A\| \mu(I)<\infty .
$$

Furthermore $\bar{f}_{A_{n+1}}(\mu) \geqq \bar{f}_{A_{n}}(\mu)$ for all $\mu \in \bar{W}$. We can therefore define

$$
\bar{f}_{\infty}(\mu)=\lim _{n \rightarrow \infty} \bar{f}_{A_{n}}(\mu)
$$

which is a nonnegative finite valued linear form on $\bar{W}$. It is easily seen that $\bar{f}_{\infty}(J)=\operatorname{trace}(J A)$ for all $J \in W$, which proves $A=A_{\bar{f}_{\infty}}$ and the lemma.

The mapping $\Phi$ is therefore an isomorphism from the set of nonnegative linear forms on $\bar{W}$ on to the set of nonnegative bounded operators. Its inverse $\Phi^{-1}$ assigns to each operator $A$ one and only one nonnegative linear form $\bar{f}_{A}$ on $\bar{W}$, and the correspondence is linear, i.e., $\bar{f}_{A+B}=\bar{f}_{A}+\bar{f}_{B}, \bar{f}_{\lambda A}=\lambda \bar{f}_{A}$ for real $\lambda \geqq 0$.

Proof of Theorem I. Let $\mu$ be a finite valued measure on the projections in $H$. We define the linear form $\bar{\mu}$ on the set of nonnegative operators by $\bar{\mu}(A)=\bar{f}_{A}(\mu)$.

Clearly $\bar{\mu}(P)=\mu(P)$ for every projection $P$ of $H$, and $\bar{\mu}$ is an exten- 
sion of $\mu$ to a nonnegative linear form on the nonnegative bounded operators. It is trivial to extend $\bar{\mu}$ to the class of all bounded operators in $H$. Let $\phi$ and $\psi$ be vectors in $H$. We define the operator $P_{\phi, \psi} n$ $=\phi(\psi, n) \quad(n \in H)$ and consider $p(\cdot, \cdot)$ defined by $p(\psi, \phi)=\bar{\mu}\left(P_{\phi, \psi}\right)$ which is clearly a complex bilinear form in $\psi$ and $\phi$. Furthermore, it is bounded because if the norm of $\phi$ is one, then $P_{\phi, \phi} \leqq I$, and $p(\phi, \phi)$ $=\bar{\mu}\left(P_{\phi, \phi}\right) \leqq \bar{\mu}(I)$. There exists therefore a bounded linear operator $J$ with $\bar{\mu}\left(P_{\phi, \psi}\right)=(\psi, J \phi)$, and since $\bar{\mu}\left(P_{\phi, \phi}\right) \geqq 0$ for all $\phi, J$ is nonnegative.

Let $\left\{\phi_{i}\right\}$ be an orthonormal basis in $H$. Then

$$
I=\sum_{i} P_{\phi_{i}, \phi_{i}}
$$

and

$$
\bar{\mu}(I)=\sum_{i} \bar{\mu}\left(P_{\phi_{i}, \phi_{i}}\right)=\sum_{i}\left(\phi_{i}, J \phi_{i}\right),
$$

which proves that trace $(J)<\infty$. Let $P_{E}$ be the projection on the closed subspace $E \subset H$, and $\left\{\psi_{i}\right\}$ an orthonormal basis in $E$. Then

$$
P_{E}=\sum_{i} P_{\psi_{i}, \psi_{i}} \cdot
$$

Noting that $\left(\psi_{i}, J \psi_{i}\right)=\operatorname{trace}\left(J^{1 / 2} P_{\psi_{i}, \psi_{i}} J^{1 / 2}\right)$, we have

$$
\begin{aligned}
\mu\left(P_{E}\right)=\bar{\mu}\left(P_{E}\right) & =\sum_{i} \bar{\mu}\left(P_{\psi_{i}, \psi_{i}}\right)=\sum_{i}\left(\psi_{i}, J \psi_{i}\right) \\
& =\sum_{i} \operatorname{trace}\left(J^{1 / 2} P_{\psi_{i}, \psi_{i}} J^{1 / 2}\right) \\
& =\operatorname{trace}\left(J^{1 / 2}\left(\sum_{i} P_{\psi_{i}, \psi_{i}}\right) J^{1 / 2}\right) \\
& =\operatorname{trace}\left(J^{1 / 2} P_{E} J^{1 / 2}\right)=\operatorname{trace}\left(J P_{E}\right),
\end{aligned}
$$

and Theorem $I$ is proved.

\section{REFERENCES}

1. A. M. Gleason, Measures on the closed subspaces of a Hilbert space, J. Math. Mech. 6 (1957), 885-893.

2. C. C. Brown, Zur Verallgemeinerung der abstrakten Integrationstheorie, $Z$. Wahrscheinlichkeitstheorie und Verw. Gebiete (4) 6 (1966), 317-348.

Mathematisches INSTITUT DER UNIVERSITÄT ERLANGEN-NÜRNBERG 